\title{
Frequency selectivity using tonal-temporal masking: Diotic vs. dichotic masking
}

\author{
WILLIAM A. YOST \\ Communication Sciences Laboratory, University of Florida, Gainesville, Florida $\mathbf{2 6 1 1}$
}

\begin{abstract}
A combined forward-backward masking procedure was used to investigate the threshold of a 30-msec, $500-\mathrm{Hz}$ signal as a function of masker frequency. The signal thresholds were obtained in two signal conditions, diotic (So) and dichotic $\left(S_{\pi}\right)$, and for two different temporal separations of the maskers. The maskers were 500 msec in duration and were presented at $75 \mathrm{~dB}$ SPL. The function relating masked signal threshold to masker frequency was used to describe frequency selectivity in the four conditions. There were no differences in frequency selectivity measured between the diotic and dichotic signal conditions and only a small difference measured between the two intermasker interval conditions. The $\mathrm{S}_{\pi}$ conditions yielded lower thresholds than did the So conditions. The change in intermasker interval from 10 to 50 msec lowered the threshold maximally $18 \mathrm{~dB}$ for the So condition and $13 \mathrm{~dB}$ for the $\mathrm{S}_{\pi}$ condition. The results indicate that in this tonal temporal masking procedure there are no differences between the diotic and dichotic critical bands.
\end{abstract}

Since Fletcher (1940) first used the concept of the "critical band" to describe the auditory system's frequency-selectivity capabilities, considerable research has been devoted to measuring critical bands. These experiments (see Scharf, 1970, for review) have involved some of the following techniques: band-narrowing (Fletcher, 1940), critical ratio (Hawkins \& Stevens, 1950), the measurement of loudness (Zwicker, Flottorp, \& Stevens, 1957), the use of frequency modulation (Scharf, 1959), and most recently, tonal masking in a variety of temporal masking paradigms (Houtgast, 1972, Note 1).

The tonal masking procedure has the advantage of measuring the width and also the shape of the critical band. The classical tone-on-tone simultaneous masking procedure of Wegel and Lane (1924) is not useful for measuring the critical band since the introduction of nonlinear distortion components (combination tones) interferes with a direct measure of frequency selectivity. However, as Houtgast has shown, if the masking tone and signal tone are separated in time, the problems of nonlinearity are eliminated. Houtgast was not the first to make this observation; others have used forward masking to study frequency selectivity (see Elliot, 1962; Glattke \& Small, 1967; Luscher \& Zwislocki, 1949). These investigators have shown that estimates of the shape of the critical bands can be obtained in temporal masking experiments.

In the tonal masking experiments, the tonal masker is viewed as establishing an interference pattern and the signal is used as a probe to investigate this

This research was supported in part by a National Institutes of Health (NIH) program project grant, an NIH training grant, an NIH research grant, a University of Florida research grant, and a grant from the Northeast Regional Data Center of the University of Florida. pattern. The interference pattern will be called the critical band. It is assumed that as the interference pattern increases, the signal level required for detection will increase. That is, the more masking being provided by the masker, the less the system is able to differentiate the frequency of the masker from that of the signal. By varying the frequency of the masker and keeping the signal frequency constant, one can measure the masked threshold of the signal as a function of masker frequency. The function relating signal threshold to masker frequency is called the critical band. The temporal masking paradigm simply prevents nonlinear combination tones from occurring when two tones of different frequencies are presented simultaneously. Therefore, tonal masking using a temporal paradigm enables direct estimation of the shape of the critical bands.

The concept of a binaural critical band has been discussed in the experimental literature on binaural beats, masking-level difference (MLD), and central masking. The results from binaural beats and MLD experiments imply that the binaural critical band is broader than that measured for monaural-type inputs. However, the data from the central masking studies indicate a small difference in frequency selectivity measured for monaural and dichotic stimuli.

The data from studies on binaural and monaural beats show that the frequency range over which binaural beats are obtained is broader than that over which monaural beats are measured (Perrott \& Nelson, 1969). Perrott and Nelson showed that best binaural beats are detected when the interaural difference in frequency $(\Delta f)$ is approximately $25-30 \mathrm{~Hz}$. As early as 1928 , Riesz had shown that best beats are detected at approximately a monaural $\Delta f$ of 3-5 Hz. Thus, Robinson (1971) and others have 
suggested that the binaural system has a broader sensitivity to frequency than does the monaural ststem.

The implications for a broad binaural critical band have been obtained from many MLD studies. The MLD is the difference betueen masked thresholds obtained for signals or maskers presented dichotically relative to those obtained when the signals and maskers are both presented diotically. The dichotic masked thresholds are smaller than diotic masked thresholds.

Some of the measures of the binaural critical bands in MLD studies are indirect. For instance, Robinson and Jeffress (1963) measured the amount of binaural masking as a function of interaural correlation of the noise at the ears. Langford and Jeffress (1964) measured the amount of binaural masking obtained as a function of varying the interaural temporal difference between the noise at the ears. Interaural temporal difference can be equated to interaural correlation if an assumption is made concerning the effective bandwidth of the noise. If, at $500 \mathrm{~Hz}$, one assumes a $100-\mathrm{Hz}$ bandwidth for the noise (a $100-\mathrm{Hz}$-wide binaural critical band), then the data of Langford and Jeffress (1964) match those of Robinson and Jeffress (1963). This implied $100-\mathrm{Hz}$ critical band is about twice the monaural critical ratio estimate at $500 \mathrm{~Hz}$ [Hawkins \& Stevens' (1950) critical ratio estimate is approximately $55 \mathrm{~Hz}$. Thus, the data imply that the binaural system has a wider critical band than does the monaural system.

Other MLD experiments have attempted to measure directly the width of the binaural critical band. Sondhi and Guttman (1966), for instance, varied the bandwidth of a section of noise in one ear which was $180^{\circ}$ out of phase with the same spectral region at the other ear. They found no. change in the binaural masking of a $500-\mathrm{Hz}$ tone until the bandwidth of the interaurally phase-inverted noise was approximately $200 \mathrm{~Hz}$; then the amount of masking increased. Thus, the data again imply that the binaural system's critical band is broader than that of the monaural system.

In the Zwislocki, Damianopoulus, Buining, and Glantz (1967) study of central masking, a tone was used at one ear to mask a signal at the other ear. Zwislocki et al. varied the frequency of the signal with the masker at $1,000 \mathrm{~Hz}$ and compared their critical band estimates to the monaural estimates of Zwicker et al. (1957). Zwislocki et al.'s binaural critical band at $1.000 \mathrm{~Hz}$ was approximately $70 \mathrm{~Hz}$, and Zwicker et al.'s was approximately $75 \mathrm{~Hz}$. Thus, Zwislocki et al.'s data indicate that dichotic stimulation can vield estimates of critical bands which are similar to monaural estimates.

Therefore. three types of psychophysical procedures (binaural beats, MLD, and central masking) yield different implications as to the width of the binaural critical band. A tone-on-tone binaural masking procedure using a temporal paradigm might help clarify these equivocal results.

\section{METHOD}

A combined forward-backward masking procedure was used to investigate the masked threshold of a $30-\mathrm{msec}, 500-\mathrm{Hz}$ tonal signal (the signal was shaped with a $10 \cdot \mathrm{msec}$ rise-decay time) placed temporally between two tonal maskers. Each masker was $500 \mathrm{msec}$ in duration (the maskers were shaped with a 10 -msec rise-decay time). In one condition. the first. or forward, masker ended approximately 5 msec before the signal began. while the second, or backward, masker began approximately 5 msec after the signal ceased $(5-\mathrm{msec} \Delta \mathrm{T}$ condition). In the other condition, the forward masker ended 25 msec before the signal began and the backward masker began $25 \mathrm{msec}$ after the signal ceased $(25-\mathrm{msec} \Delta \mathrm{T}$ condition).

In the diotic condition (So). the maskers and signal were presented exactly the same to both ears. In the dichotic condition $\left(S_{n}\right)$. the maskers were presented the same to both ears, but the signal was presented to the right ear $180^{\circ}$ out of phase relative to the left ear. There were no other waveform differences measured at the output of the TDH -39 headphones.

The frequency of the maskers varied from 250 to $1,000 \mathrm{~Hz}(250$. $325,425,475,500,525,550,575,600,625,700$, and $1,000 \mathrm{~Hz}$ ). and the maskers were presented at $75 \mathrm{~dB}$ SPL. The signal frequency was always $500 \mathrm{~Hz}$. A two-alternative. forced-choice (2AFC) adaptive procedure was used to determine the masked threshold of the signal. The procedure was the one-up, two-down procedure described by Levitt (1971). That is, each time the observer was incorrect in identifying which interval contained the signal (the signal occurred in the first observation interval with a probability of 0.5 ), the signal level was increased by $2 \mathrm{~dB}$. The signal level was decreased by $2 \mathrm{~dB}$ whenever the observer was correct tuice in a row. This procedure was used to estimate the signal level that would be required for a percent correct $[P(C)]$ of approximately $70 \%$ in a $2 \mathrm{AFC}$ task. Data collection was stopped after 12 reversals in the level of the signal. The threshold for one of these runs was calculated by obtaining the midpoint of the range of intensities associated with each reversal in the signal level. The mean of the last nine (out of the possible 12) midpoint intensities was used to define the threshold for any run. The mean threshold calculated over three runs was used to determine the masked signal threshold for any one condition. Between 120 and $2002 \mathrm{AFC}$ trials were required for each condition to obtain these thresholds. In addition to the masked threshold determinations, the same 2AFC adaptive procedure was used to obtain the absolute threshold for the 30 -msec. $500-\mathrm{Hz}$ signal in both the $S_{0}$ and $S_{\pi}$ conditions.

The adaptive procedure was controlled by a PDP-8/e computer and a digitally controlled attenuator. Two trained observers. one male and one female, with normal hearing served as paid observers. The observers listened in a single-walled IAC booth for $2 \mathrm{~h}$ per day over a 2-month period.

\section{RESULTS}

The results in Figures 1-3 are plotted as the energy of the signal at masked threshold as a function of masker frequency. The data are shown for the So and $S_{\pi}$ conditions and for both signal-masker temporal differences. The lines drawn at the bottom of the figures represent the absolute thresholds for the $30-\mathrm{msec} 500-\mathrm{Hz}$ signal in both the So and $S_{\pi}$ conditions. Figure 1 represents Subjects 1's data, Figure 2 represents Subject 2's data, and Figure 3 gives the average data. 


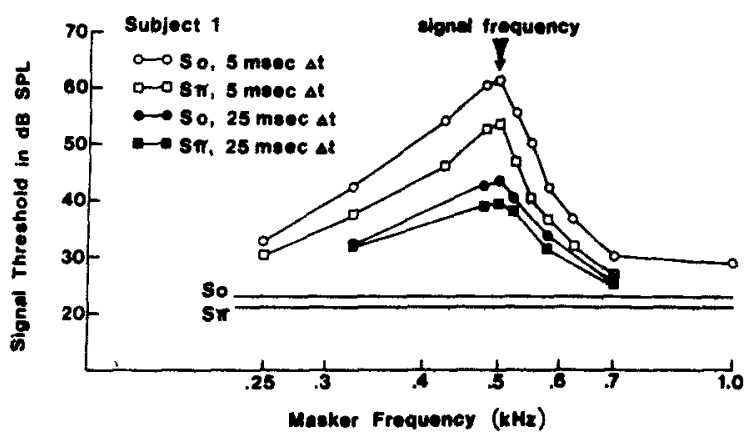

Figure 1. The makred threshold of the aignal in dB SPL as a function of the malke frequency. The arrow hadicates that the signal frequency was $500 \mathrm{~Hz}$. The $(\mathrm{O}-\mathrm{O})$ symbole represent the So and 5-muec $\Delta T$ condition, the $(\square-\square)$ aymbols represent the $S_{h}$ and $t$-meec $\Delta T$ condition, the $(\bullet-\infty)$ aymbole represent the So and 25-maec $\Delta T$ condition, and the $\left(\square-D\right.$ ) anmbole represent the $S_{n}$ and 25-minec $\Delta T$ condition. The solld lhew at the bottom reprenent the absolute thresholds for the So and $S_{n}$ slenals. Thi figure show the data for Subject 1.

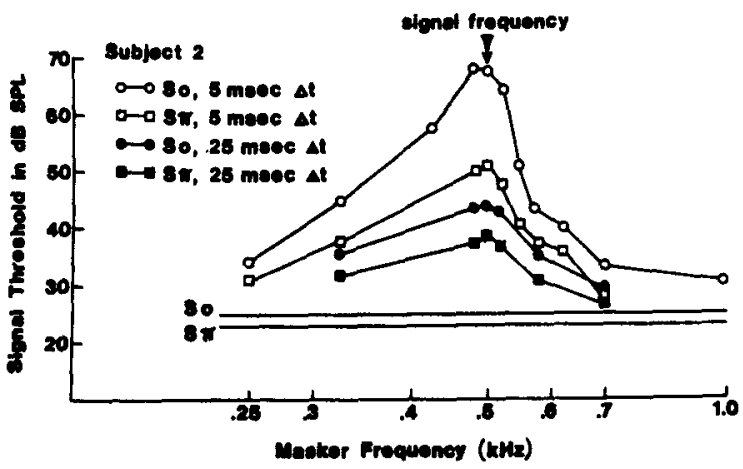

Figure 2. The data are plotted exactly the ame as in Figare 1. This figure repreaents the data for Subject 2.

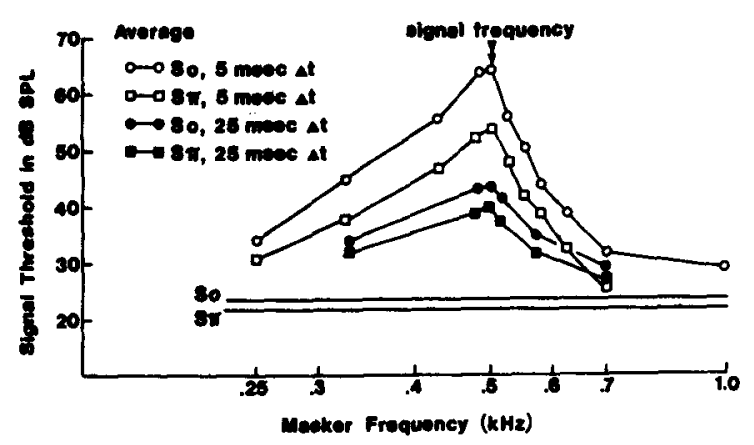

Figure 3. The data are plotted exactly the came an in Flezure 1. This figure represent the mean dati areraged orer Sabject 1 (Figure 1) and Subject 2 (Figare 2).

Table 1 describes approximate estimates of the bandwidths and the approximate estimates of the decibel per octave roll-off for the low- and high-frequency sides of each of the four functions for the average observer. The bandwidths were determined at the points $3 \mathrm{~dB}$ down from the maximum threshold at the masker frequency of $500 \mathrm{~Hz}$. The low-frequency roll-off was computed over the range from 325 to $500 \mathrm{~Hz}$, and the high-frequency roll-off was computed over the range from 500 to $625 \mathrm{~Hz}$ for the 5 -msec $\Delta \mathrm{T}$ conditions and from 500 to $575 \mathrm{~Hz}$ for the $25-\mathrm{msec} \Delta \mathrm{T}$ conditions.

Table 2 describes for the average subject the decibel difference between the So and $S_{\pi}$ conditions for the two $\Delta T$ conditions. As can be seen, the largest differences exist when the masker frequency equaled the signal frequency; the differences then decrease slightly, with some irregularities, as the masker frequency was separated-from the signal frequency. These data are essentially the masking-level differences (MLD) at each of the masker frequencies.

The average decibel difference between the 5-msec $\Delta T$ and the 25-msec $\Delta T$ conditions is shown for the So and $S_{\pi}$ functions in Table 3 . The differences are largest for the So condition and when the masker frequency equals the signal frequency.

Table 1

Estimates of the Bandwidths in Hertz and the Low- and HighFrequency Roll-Offs in Decibels Per Octave for Each of the Four Conditions for the Average Subject

\begin{tabular}{lcccc}
\hline & \multicolumn{2}{c}{$5 \mathrm{msec}, \Delta \mathrm{T}$} & \multicolumn{2}{c}{$25 \mathrm{msec}, \Delta \mathrm{T}$} \\
& So & $\mathrm{S}_{\pi}$ & So & $\mathrm{S}_{\boldsymbol{\pi}}$ \\
\hline Bandwidth (Hz) & 45 & 60 & 75 & 80 \\
$\begin{array}{l}\text { Low-Frequency Roll-Off } \\
\text { (dB/octave) }\end{array}$ & 36 & 28 & 18 & 16 \\
$\begin{array}{l}\text { High-Frequency Roll-Off } \\
\text { (dB/octave) }\end{array}$ & 123 & 113 & 112 & 107 \\
\hline
\end{tabular}

Table 2

Difference in Decibels for the Average Subject Between the So and $S_{\pi}$ Conditions

\begin{tabular}{ccc}
$\begin{array}{c}\text { Masker Frequency } \\
(\mathbf{H z})\end{array}$ & $\begin{array}{c}5 \mathrm{msec}, \Delta \mathbf{T} \\
(\mathrm{dB})\end{array}$ & $\begin{array}{c}25 \mathrm{msec}, \Delta \mathbf{T} \\
(\mathrm{dB})\end{array}$ \\
\hline 250 & 3 & \\
325 & 6 & 1 \\
425 & 10 & \\
475 & 12 & 2 \\
500 & 12 & 4 \\
525 & 9 & 3 \\
550 & 9 & 2 \\
575 & 5 & 1 \\
625 & 7 & 1 \\
700 & 6 & \\
\hline
\end{tabular}

Table 3

Decibel Difference for the Average Subject Between the 5-msec, $\Delta T$ Condition and the 25-msec, $\Delta T$ Conditions:

\begin{tabular}{ccc}
$\begin{array}{c}\text { Masker Frequency } \\
(\mathrm{Hz})\end{array}$ & $\begin{array}{c}\text { So } \\
\text { (dB) }\end{array}$ & $\begin{array}{c}\mathrm{S}_{\pi} \\
(\mathrm{dB})\end{array}$ \\
\hline 325 & 10 & 5 \\
475 & 18 & 12 \\
500 & 18 & 13 \\
525 & 15 & 10 \\
575 & 10 & 6 \\
700 & 1 & 1 \\
\hline
\end{tabular}




\section{DISCUSSION}

The results from this study indicate that, at least in this temporal masking paradigm, there are no differences between diotic and dichotic critical bands. Neither the shapes nor the bandwidths are different for the two binaural conditions. Although there were some changes in frequency selectivity between the 5-msec $\Delta \mathrm{T}$ and the $25-\mathrm{msec} \Delta \mathrm{T}$ conditions, this did not result in a difference between the diotic and dichotic critical bands measured at each of these two signal-masker temporal separations. Thus, the similarity in frequency selectivity obtained between the diotic and dichotic conditions probably does not depend to a great extent on the temporal separation between the signal and the maskers. The relatively large MLDs do indicate that the $S_{\pi}$ condition is different from the So condition, and thus we are probably probing binaural processing with the $S_{\pi}$ condition.

The bandwidths and shapes of the frequency selectivity functions obtained in the 5 -msec $\Delta T$ condition agree with those obtained by Houtgast (Note 1). Houtgast used a variety of temporal masking conditions and showed that the bandwidths and shapes of the frequency selectivity functions were similar to single-unit tuning curves obtained from the auditory nerve.

We obtained approximately a 2 -dB difference in absolute detection of the $30-\mathrm{msec}$ signal between the So and $S_{\pi}$ conditions. This agrees reasonably well with the 1-dB difference measured by Jeffress and Diercks (1962).

The masked threshold in the So condition decreased approximately $18 \mathrm{~dB}$ from the 5 -msec $\Delta \mathrm{T}$ condition (intermasker interval of $10 \mathrm{msec}$, not including the signal) to the $25 \cdot \mathrm{msec} \Delta \mathrm{T}$ condition (intermasker interval of $50 \mathrm{msec}$, not including the signal) at a masker frequency of $500 \mathrm{~Hz}$. Robinson and Pollock (1973), using a combined forwardbackward masking procedure, found that the noise-masked threshold of a click decreased approximately $15 \mathrm{~dB}$ as the intermasker temporal interval increased from 10 to $50 \mathrm{msec}$. Thus, there is some agreement between the tonal-temporal masking and noise-click temporal masking data in regard to the decrease in threshold as a function of increases in the intermasker interval.

The major difference between the 5-msec $\Delta T$ and 25 -msec $\Delta \mathrm{T}$ conditions is in the low-frequency roll-offs in both the So and $S_{\pi}$ conditions. The low-frequency roll-offs in the $25-\mathrm{msec} \Delta \mathrm{T}$ condition are approximately half as steep as those obtained in the 5-msec $\Delta T$ condition. This change in the low-frequency attenuation rate causes the bandwidths in the $25-\mathrm{msec} \Delta \mathrm{T}$ condition to be approximately one-third again as large as those in the $5-\mathrm{msec} \Delta T$ condition. We have no explanation as to why only the low-frequency masker would mask a high-frequency signal differently depending on the temporal separation between the signal and maskers. Exact measurements of the slope of the frequency selectivity functions are difficult to obtain. This is especially true when there is very little masking, such as in the 25-msec $\Delta T S_{\pi}$ condition. A change of a few decibels in masking can yield a rather larger change in the slope estimates. Thus, these data must be interpreted as indicating only the relative differences between slopes.

In the present study, the masker and signal were diotic with respect to frequency. This is not the case for the binaural beat experiments which indicate broad binaural critical bands. Thus, one might obtain a difference in frequency selectivity between the So and $S_{\pi}$ conditions in a temporal masking study if either the signal or masker were presented dichotically with respect to frequency. In the MLD studies of Langford and Jeffress (1964), Robinson and Jeffress (1963), and Sondhi and Guttman (1966), the masker was dichotic in time or phase and the signal, diotic. In this experiment, the signal was dichotic and the masker, diotic. Since the investigators mentioned above measured large dichotic critical bands, a frequency selectivity difference between the So and $S_{\pi}$ conditions might be obtained in a tonal-temporal masking procedure if the masker were presented dichotically in time or phase. That is, in the MLD nomenclature, if the conditions had been $M_{\pi}$ So and MoSo rather than $\mathrm{MoS}_{\pi}$ and MoSo, there might have been a difference in the critical band estimates. The $\mathrm{M}_{\pi}$ So condition was not used, since Yost, Nielsen, Tanis, and Bergert (1974) showed that in a simultaneous tonal masking experiment very small MLSs (less than $4 \mathrm{~dB}$ ) were measured between the $\mathrm{M}_{\pi}$ So and MoSo conditions. Thus, it appears that there are no differences in frequency selectivity between the MoSo and $\operatorname{MoS}_{\pi}$ conditions when a combined forward-backward masking procedure is used.

\section{REFERENCE NOTE}

1. Houtgast, T. Psychophysical experiments on "tuning curves" and "tone-tone inhibition." Institute for Perception RVO-TNO Report No. IZF 1972-25, 1972.

\section{REFERENCES}

ElLIOTT. L. Backward and forward masking of probe tones of different frequencies. Joumal of the Acoustical Society of America, 1962, 34, 8.

Fletcher, H.. Auditory patterns. Review of Modern Physics. $1940,12,47-65$.

GlattKe. T.. \& Small, A. Frequency selectivity of the ear in forward masking. Journal of the Acoustical Society of America. $1967,42,154-157$.

Hawkins, J. E., \& Stevens. S. S. The masking of pure tones of speech by white noise. Joumal of the Acoustical Society of America. 1950, 22, 6-13. 
Houtcast, T. Psychophysical evidence for lateral inhibition in hearing. Joumal of the Acoustical Society of America, 1972, 51, 1885-1894.

JefrRess, L. A., \& DizRcks, K. J. Interaural phase and the absolute threshold for tone. Joumal of the Acoustical Society of America, $1962,34,981-984$.

LANGFoRd, T. L., \& JefrRess, L. J. Effect of noise cross correlation on binaural signal detection. Joumal of the Acoustical Society of America, 1964, 36, 1455-1458.

LevrT, H. Transformed up-down methods in psychoscoustics. Journal of the Acoustical Society of America, 1971, 49, 467-477.

LUSCHER, E., \& ZwisLOCKI, I. The decay of sensation and the remainder of adaptation after short-pure-tone impulses on the ear. Joumal of the Acoustical Society of America, 1949, 21. 135.

Perrott, D. R., \& Nel.son, M. R. Limits for detection of binaural beats. Journal of the Aconstical Society of America, 1966, 46, $1477-1481$.

RoBINson, D. E. The effect of interaural signal-frequency disparity on signal detectability. Joumal of the Acoustical Society of America, 1971, 50, 568-572.

Ronisson, D. E., \& JefFress, L J. Effect of varying interaural noise correlation on the detectability of tone signals. Journal of the Acoustical Society of America, 1963, 35, 1947-1952.

Robinson, C. E., \& Pollack, I. Interaction between forward and backward masking: A measure of integrating period of the auditory system. Journal of the Acoustical Society of America, 1973, 53, 1313-1317.

SCRARF, B. Loudness of complex sounds as a function of the number of components. Joumal of the Acoustical Socicty of America, 1959, 31, 783-785.

Scharf, B. Critical bands. In J. Tobias (Ed.), Foundation of modem auditory theory (Vol. 1). New Yort: Academic Press, 1972.

SoNDni, M. M., \& GuTmun, N. Width of spectrum effective in the binaural release from masking. Journal of the Acoustical Society of America, 1966, 40,600-607.

Yost, W. A., Nlelsex, D. W., Tans, D. C., \& Beggext, B. Tone-on-tone binaural masking with an autiphasic masker. Perception \& Psychophysics, 1974, 15, 233-237.

Zwicker, E., Flottorp, G., \& Stevens, S. S. Critical bandwidth in loudness summation. Journal of the Acoustical Society of America, 1957, 30, 85-92.

Zwislock, J. J., Damunopoulos, E. N., Bunnwe, E., \& Glantz, J. Central masking: Some steady-state and transient effects. Perception \& Psychophysics, 1967, 2, 59-64.

(Received for publication April 18, 1975; accepted August 1, 1975.) 